\title{
Brain-Controlled Functional Electrical Stimulation for Lower-Limb Motor Recovery in Stroke Survivors
}

\author{
Colin M. McCrimmon*1, Christine E. King*1, Po T. Wang ${ }^{1}$, Steven C. Cramer ${ }^{2}$, Zoran Nenadic ${ }^{1,3}$, An H. Do ${ }^{2}$
}

\begin{abstract}
Despite the prevalence of stroke-induced gait impairment due to foot drop, current rehabilitative practices to improve gait function are limited, and orthoses can be uncomfortable and do not provide long-lasting benefits. Therefore, novel modalities that may facilitate lasting neurological and functional improvements, such as brain-computer interfaces (BCIs), have been explored. In this article, we assess the feasibility of BCI-controlled functional electrical stimulation (FES) as a novel physiotherapy for post-stroke foot drop. Three chronic stroke survivors with foot drop received three, 1-hour sessions of therapy during 1 week. All subjects were able to purposefully operate the BCI-FES system in real time. Furthermore, the salient electroencephalographic (EEG) features used for classification by the data-driven methodology were determined to be physiologically relevant. Over the course of this short therapy, the subjects' dorsiflexion active range of motion (AROM) improved by $3^{\circ}, 4^{\circ}$, and $8^{\circ}$, respectively. These results indicate that chronic stroke survivors can operate the BCI-FES system, and that BCI-FES intervention may promote functional improvements.
\end{abstract}

\section{INTRODUCTION}

With over 795,000 new cases each year, stroke remains the leading cause of long-term disability in the US [1]. It is estimated that $30-60 \%$ of the 7 million stroke survivors in the US suffer from long-term gait deficits, primarily due to foot drop [2]. However, current gait therapies for stroke survivors have limited benefits once these individuals reach their rehabilitative plateau 6-8 months post-stroke [3], and assistive devices, such as ankle-foot orthoses, have no lasting effects after removal. Hence, novel therapies that promote lasting neurological and functional improvements are needed. Brain-computer interface (BCI) controlled functional electrical stimulation (FES) may be one such approach [4].

BCIs use computers to translate signals of the central nervous system into control commands for external devices. They may also promote motor relearning in stroke survivors through Hebbian-like neuroplastic changes [5]. More specifically, coactivation of the post-infarct cortex (via the BCI) and peripheral neuromuscular system (i.e. antidromic excitation of alpha motor neurons via FES) may lead to long-term synaptic potentiation between these upper and lower motor neurons. A preliminary study by Daly et al. [6] suggests

\footnotetext{
${ }^{*}$ These authors contributed equally.

This work was funded by the Institute of Clinical and Translational Science at UCI, and the American Academy of Neurology.

${ }^{1}$ Dept. of Biomedical Eng., UCI, Irvine, CA 92697 USA \{ cmccrimm, kingce, ptwang, znenadic\}@uci.edu

${ }^{2}$ Dept. of Neurology, UCI, Irvine, CA 92697 USA andeuci.edu

${ }^{3}$ Dept. of Electrical Eng. and Comp. Sci., UCI, Irvine, CA 92697 USA
}

that only a few sessions of finger and upper-limb BCIFES therapy may lead to lasting improvements in poststroke motor function. Motivated by this work, we tested the feasibility of using a BCI-FES system to treat stroke-induced foot drop. The present study represents an extension of our previous work to a population of chronic stroke survivors.

\section{Methods}

\section{A. Overview and Subject Recruitment}

Chronic stroke subjects participated in 3 daily sessions of foot-drop-targeted BCI-FES therapy over the course of 1 week using a previously developed system [4], [7] (details in Fig. 1). During each session, a subject-and-day specific decoding model that classified idling and dorsiflexion states from electroencephalographic (EEG) data was created before performing several online runs, in which the system detected the subject's intention to dorsiflex or idle and controlled FES delivery, appropriately, in real time. Subjects' dorsiflexion active range of motion (AROM) was measured at the beginning and end of the study.

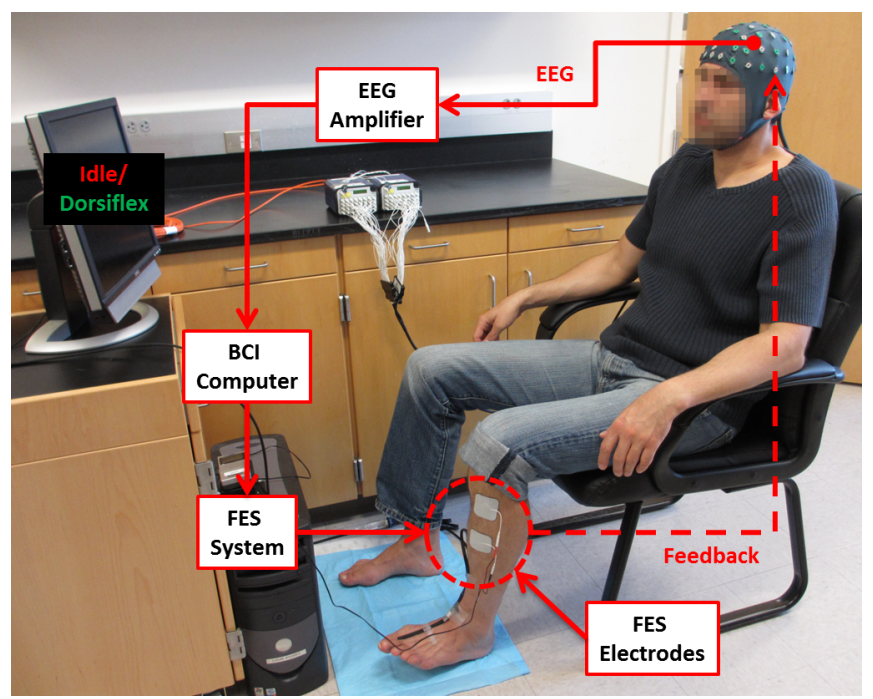

Fig. 1. A representative setup of the BCI-FES system. In response to computerized cues, the subject attempts idling or dorsiflexion, resulting in EEG changes that are detected by the BCI computer. The computer then instructs the FES system to either deliver or cease electrical stimulation respectively, providing feedback to the subject.

This study was approved by the University of California, Irvine Institutional Review Board. Inclusion criteria were 
chronic adult stroke survivors ( $>6$ months post-stroke) exhibiting moderate to severe foot drop; exclusion criteria were the lack of FES response, presence of electronic implants, and severe ankle contractures or spasticity.

\section{B. Offline Training and Decoding Model Generation}

At the start of each session, EEG data were recorded from subjects using a 63-channel cap as they followed 100 alternating 6-s-long computerized cues of idling and attempted dorsiflexion on the paretic foot. The offline training data were then used to generate a subject-and-day specific decoding model using the methodologies described in [4] and [7]. Briefly, those channels with excessive artifacts were removed in an automated manner (details in [8]), and $2 \mathrm{~s}$ of transition time were removed from each idling/dorsiflexion epoch. Power spectral densities (PSDs, in $2 \mathrm{~Hz}$ bins from 8$30 \mathrm{~Hz}$ ) were then calculated for each of the resulting 4-s-long trials, and the PSDs were projected on two lower dimensional subspaces that retain the class differences using class-wise principal component analysis (CPCA) [9]. This was followed by either linear discriminant analysis (LDA) or approximate information discriminant analysis (AIDA) [10], depending on which method yielded better offline performance. Specifically, if $\Phi_{\mathrm{CPCA}}: \mathbb{R}^{C \times F} \rightarrow \mathbb{R}^{n}$ is a piecewise linear CPCA mapping, where $n<C \times F$ (C-number of retained EEG channels, $F$-number of frequency bins), and $M_{\mathrm{DA}}: \mathbb{R}^{n} \rightarrow \mathbb{R}^{1}$ is either the LDA or AIDA linear transform, then feature extraction is described by:

$$
f^{*}=M_{\mathrm{DA}} \Phi_{\mathrm{CPCA}}\left(x^{*}\right)
$$

Here, $x^{*} \in \mathbb{R}^{C \times F}$ is an observation in the original sample space and $f^{*} \in \mathbb{R}^{1}$ is its 1-D representation. Classification of $x^{*}$ was then performed using the following Bayesian classifier:

$$
x^{*} \in \begin{cases}I, & \text { if } P\left(I \mid f^{*}\right) \geq P\left(D \mid f^{*}\right) \\ D, & \text { otherwise }\end{cases}
$$

Here, $P\left(I \mid f^{*}\right)$ and $P\left(D \mid f^{*}\right)$ represent the posterior probabilities of idling and dorsiflexion, respectively, given the observed feature, $f^{*}$. Feature extraction and classification were tested using stratified 10-fold cross validation before using the decoding model for real-time classification of the subject's intention (i.e. idling or dorsiflexion) during subsequent online runs.

\section{Calibration}

To avoid noisy transitions, the system was implemented as a binary state machine (BSM) (Fig. 2) with state transition thresholds $T_{I}$ and $T_{D}$. These values were manually chosen using each state's posterior probability distribution (details in [4] and [7]), and if necessary, were adjusted between online runs to maximize the performance of the system during real-time operation.

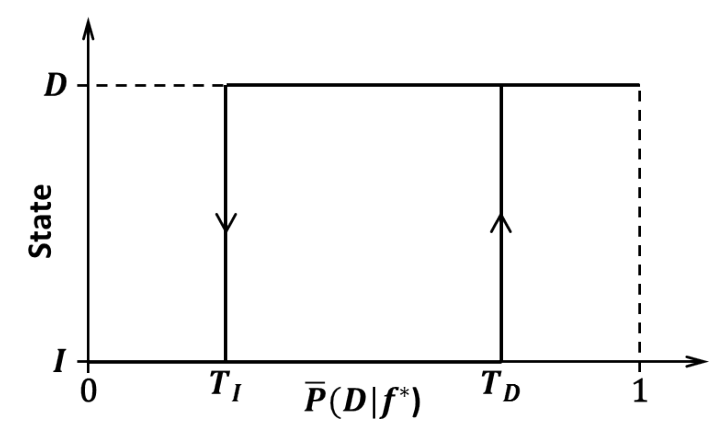

Fig. 2. Hysteresis of the BSM. The system transitions from the idling to dorsiflexion state when $\bar{P}\left(D \mid f^{*}\right)>T_{D}$, and from the dorsiflexion to idling state when $\bar{P}\left(D \mid f^{*}\right) \leq T_{I}$. When $T_{I} \leq \bar{P}\left(D \mid f^{*}\right) \leq T_{D}$, the system remains in the current state. $\bar{P}\left(\bar{D} \mid f^{*}\right)$ is the average posterior probability as described in Section II-D.

\section{BCI-FES Therapy}

Before initiating the therapy, surface FES electrodes were placed over the proximal deep peroneal nerve on the paretic leg, and stimulation parameters were adjusted to achieve $\sim 15^{\circ}$ of dorsiflexion without causing discomfort. During each 1-hour-long therapy session, subjects performed as many online BCI-FES runs as possible. Each online run consisted of 10 alternating 10-s-long epochs of "Idle" and "Dorsiflex" demarcated by computerized cues. As described in [4] and [7], the BCI-FES system detected the subjects' intention to dorsiflex or idle from their EEG signals as follows. Posterior probabilities were calculated from a 0.75 s sliding window of EEG data using the decoding model, and were averaged over the most recent 1.5-2.0 s using a sliding window (yielding $\bar{P}\left(D \mid f^{*}\right)$ ). State transitions were then initiated using the BSM described in Fig. 2. To this end, the BCI delivered FES whenever the system detected a dorsiflexion state, and discontinued FES when it detected an idling state. To assess the performance of the system during each online run, maximum cross-correlation coefficients between the $\mathrm{BCI}$ state and computer cues were calculated, and corresponding significances were determined by 10,000 Monte Carlo simulations (described in [11]). Note that averaging can introduce a time delay for state transitions, so correlations at non-zero lag had to be considered. Ultimately, each subject participated in a total of 3 daily sessions over the course of a week, and their dorsiflexion AROM was measured 3 times before and after the study (by a single, non-blinded rater) using standard goniometry.

\section{E. Analysis of Decoding Model}

To determine if the frequencies and brain areas with large CPCA-AIDA weights were physiologically relevant to foot dorsiflexion, the $C \times F$ weights were compared to signal-tonoise ratios (SNRs) using the following analysis. The SNR was calculated as:

$$
\operatorname{SNR}\left(x_{i, j}\right)=\frac{\left(\mu_{I}\left(x_{i, j}\right)-\mu_{D}\left(x_{i, j}\right)\right)^{2}}{\sigma_{I}^{2}\left(x_{i, j}\right)+\sigma_{D}^{2}\left(x_{i, j}\right)} ; i=1, \ldots, C ; j=1, \ldots, F
$$

where $x_{i, j}$ are the observations at channel $i$ and frequency $j$, and $\mu_{I}$ and $\sigma_{I}$ and $\mu_{D}$ and $\sigma_{D}$ are the mean and standard 


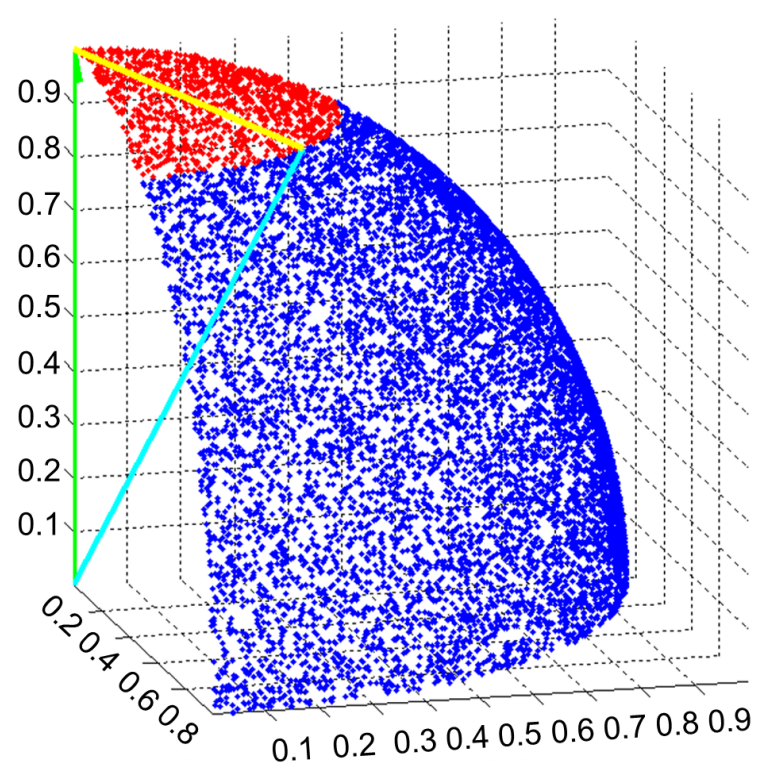

Fig. 3. An example of the comparative analysis performed between a 3-D SNR vector (green), $\vec{g}$, and a $3-\mathrm{D}$ feature extraction vector (cyan), $\vec{c}$. The yellow vector $(\vec{y})$ represents the difference between the feature extraction and SNR vectors. The Monte Carlo generated points that fell within a distance of $\|\vec{y}\|$ to $\vec{g}$ are red, while those that fell outside are blue. These points are uniformly distributed over the positive region (octant) of a 3-D sphere.

deviation of the idle and dorsiflexion class observations respectively at each channel and frequency (see [7] for details). Then, to assess how disparate the extracted features were from the SNRs, both were vectorized and scaled to unit length, and the $L^{2}$-norm (Euclidean distance) of their vector difference was calculated. Note that this metric is often used for quantifying image similarity [12]. Significance ( $p<0.001)$ was determined by 100,000 Monte Carlo simulations using a uniform distribution across the positive region of a $C \times F$ dimensional hyperspherical surface (with unit radius). Fig. 3 provides a simplified (3-D) example of this comparative analysis.

\section{RESULTS}

Three chronic stroke survivors with foot drop achieved purposeful operation of the BCI-FES system using physiologically relevant brain areas and frequencies, and all had a possible increase in dorsiflexion AROM after only 3 sessions. Their demographic data and offline/online performance results are summarized in Table I. Note that no adverse effects were observed during or after the BCI-FES therapy. In addition, the EEG decoding models for all subjects and sessions performed well above the chance level (50\%), allowing for purposeful online operation of the BCI-FES system. Furthermore, the models' features (1) exhibited a high degree of consistency with spatio-spectral physiological data, as measured by the SNRs (3). A representative example of this similarity, using subject S1's $1^{\text {st }}$ session, is provided in Fig. 4. Quantitatively, the feature extraction and SNR vectors were found to be significantly similar for all subjects and sessions except for subject S1's $2^{\text {nd }}$ session. Finally, cross-correlation analysis of the online runs revealed that all subjects had purposeful real-time control (Table I). More importantly, all subjects exhibited improved dorsiflexion AROM after the short-term therapy (Table I).

\section{Discussion}

While the study was limited in sample size, the results provide preliminary evidence that BCI-FES physiotherapy may be effective in treating post-stroke foot drop. With the exception of one daily session, the system demonstrated its ability to use relevant brain areas and frequencies that underlie physiological control of attempted foot dorsiflexion and idling in the post-stroke cortex. For example, the decoding model generated for subject S1's $1^{\text {st }}$ session matched the SNR map, and utilized the classical foot representation area, as well as posteriorly expanded brain areas, to distinguish between idling and attempted dorsiflexion states (Fig. 4). In the single daily session (subject $S 1$ 's $2^{\text {nd }}$ session) where the extracted features did not significantly match the SNRs, the data-driven methodology extracted the expected features, but excessive noise at other frequencies and brain areas changed the SNR map such that the analysis resulted in insignificance. Overall, these results indicate that our feature extraction and classification techniques are able to detect relevant upper motor neuron areas and frequencies, and by coupling this to lower motor neuron stimulation, our BCI-FES therapy may be able to elicit positive Hebbian-like neuroplastic changes.

All 3 subjects seemed to demonstrate improvements in their dorsiflexion AROM after only 3 daily sessions. Although these improvements surpassed our criterion for measurable change $\left(2.5^{\circ}\right)$ [13], other authors have suggested a larger day-to-day error in dorsiflexion goniometric measurements $\left(\leq 4^{\circ}\right)[14]$. Even still, S3 showed improvement. This is intriguing because the subjects were considered to have reached a rehabilitative plateau, as they were all $\geq 9$ months post-stroke and had already received standard physiotherapy prior to the study. Furthermore, subject S1 was a longterm user of a commercial FES system for foot drop (L300, Bioness, Valencia, CA), so the observed improvements are unlikely to be solely due to the use of FES. Thus, preliminary evidence suggests that dorsiflexion BCI-FES therapy does facilitate beneficial neuroplastic changes. However, further studies are required to determine whether these changes translate into lasting functional gait improvements.

\section{CONCLUSION}

Three chronic stroke survivors underwent BCI-FES therapy for 3 daily 1-hour sessions. The results suggest that all subjects gained purposeful real-time control of the BCI-FES system, and that the frequencies and brain areas utilized by the feature extraction techniques were consistent with physiological findings (expressed as SNR). Moreover, some, if not all, of the subjects exhibited improvements in dorsiflexion AROM. Hence, this BCI-FES therapy holds promise as a new modality to treat post-stroke foot drop. 
TABLE I

SUMMARY OF THE DEMOGRAPHIC DATA AND PERFORMANCE RESULTS.

\begin{tabular}{lccllllll}
\hline Subject & Age & $\begin{array}{l}\text { Time from } \\
\text { stroke }(\mathrm{mo})\end{array}$ & $\begin{array}{l}\text { Clinical presentation } \\
\text { (right=R, left=L) }\end{array}$ & $\begin{array}{l}\text { \# of } \\
\text { channels } \\
\text { used }\end{array}$ & $\begin{array}{l}\text { Average offline } \\
\text { decoding model } \\
\text { accuracy }(\%)\end{array}$ & $\begin{array}{r}\text { Total \# of } \\
\text { BCI-FES runs } \\
\text { completed }\end{array}$ & $\begin{array}{l}\text { Cross-correlation } \\
\text { between cues and } \\
\text { BCI-FES response }\end{array}$ & $\begin{array}{l}\text { Dorsiflexion } \\
\text { AROM }\left({ }^{\circ}\right) \\
(\text { before/after) }\end{array}$ \\
\hline $\mathrm{S} 1^{\dagger}$ & 60 & 25 & hemiparesis (R) & 63 & $97.9 \pm 1.4$ & 20 & $0.439(\mathrm{p}<0.001)$ & $5 / 8$ \\
\hline $\mathrm{S} 2$ & 83 & 29 & hemiparesis (R) & 32 & $93.3 \pm 4.7$ & 25 & $0.659(\mathrm{p}<0.001)$ & $3 / 7$ \\
\hline $\mathrm{S} 3$ & 59 & 9 & hemiparesis (L) & 32 & $90.9 \pm 1.3$ & 12 & $0.629(\mathrm{p}<0.001)$ & $0 / 8$ \\
\hline
\end{tabular}

† Data from this subject have been previously presented in [4].

Subjects S2 and S3 used a 32-channel subset of EEG electrodes during the each daily session to mitigate motion and electromyogram artifacts.

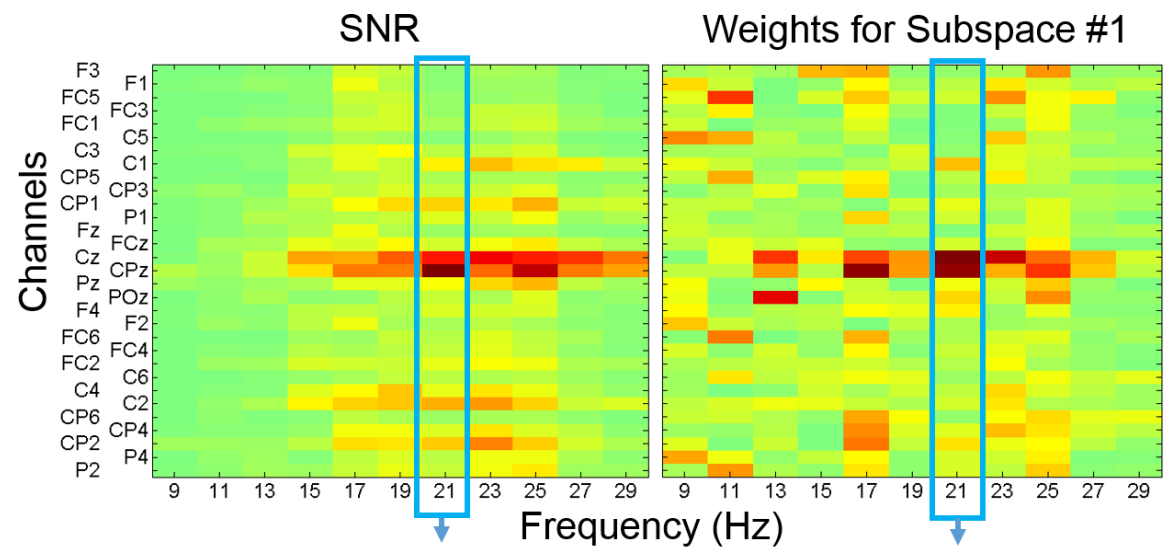

Weights for Subspace \#2

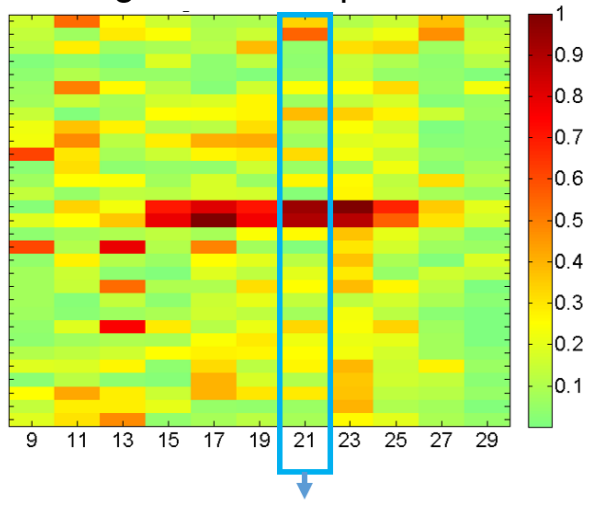

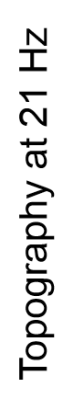
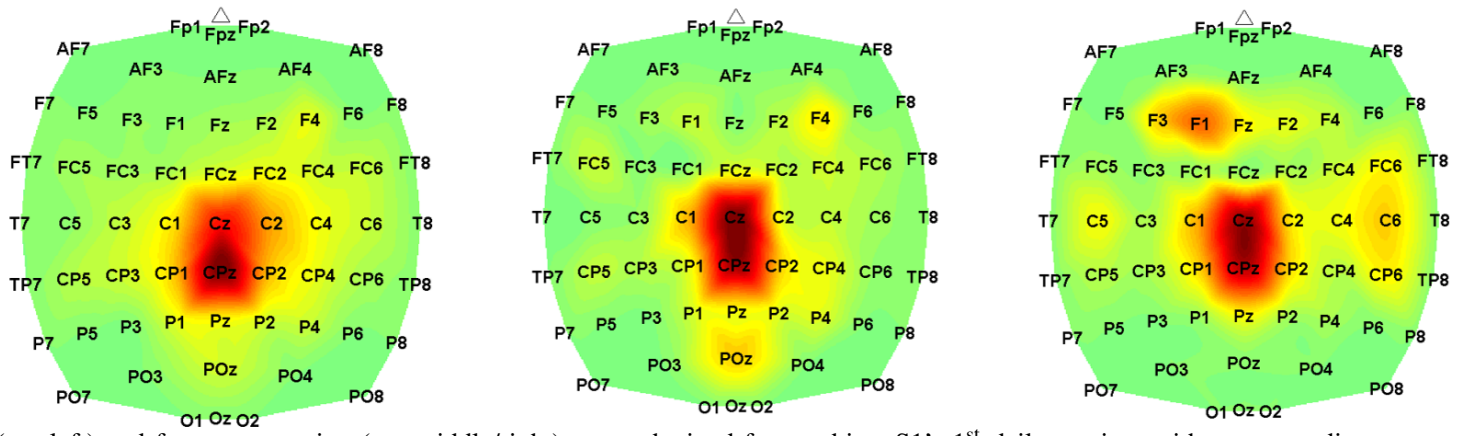

Fig. 4. SNR (top left) and feature extraction (top middle/right) maps obtained from subject S1's $1^{\text {st }}$ daily session, with corresponding topographic maps at 20-22 Hz. Only a 31 channel subset is displayed to enhance clarity. Note that the subject predominantly utilized the beta band at $20-22 \mathrm{~Hz}$, and these features were most prominent over channels $\mathrm{Cz}$ and $\mathrm{CPz}$, possibly due to stroke-induced posterior expansion of the foot representation area.

\section{REFERENCES}

[1] V. L. Roger et. al, "Heart disease and stroke statistics-2012 update: a report from the American Heart Association," Circulation, vol. 125, no. 1, pp. e2-e220, 2012.

[2] H. S. Jørgensen, H. Nakayama, H. O. Raaschou, and T. S. Olsen, "Recovery of walking function in stroke patients: the Copenhagen stroke study," Arch Phys Med Rehab, vol. 76, no. 1, pp. 27-32, 1995.

[3] P. Duncan and S. Lai, "Stroke recovery," Top Stroke Rehabil, vol. 4, no. 3, pp. 51-58, 1997.

[4] A. H. Do, P. T. Wang, C. E. King, A. Schombs, S. C. Cramer, and Z. Nenadic, "Brain-computer interface controlled functional electrical stimulation device for foot drop due to stroke," in Proc 34th Ann Int Conf IEEE Eng Med Biol Soc, pp. 6414-6417, 2012.

[5] D. N. Rushton, "Functional electrical stimulation and rehabilitation-an hypothesis," Med Eng Phys, vol. 25, no. 1, pp. 75-78, 2003.

[6] J. J. Daly et al., "Feasibility of a new application of noninvasive brain computer interface (BCI): a case study of training for recovery of volitional motor control after stroke," J Neurol Phys Ther, vol. 33, no. 4, pp. 203-211, 2009.

[7] A. H. Do, P. T. Wang, C. E. King, A. Abiri, and Z. Nenadic, "Braincomputer interface controlled functional electrical stimulation system for ankle movement," J Neuroeng Rehabil, vol. 8, no. 49, 2011.
[8] P. T. Wang, C. E. King, L. A. Chui, A. H. Do, and Z. Nenadic, "Self-paced brain-computer interface control of ambulation in a virtual reality environment," J Neural Eng, vol. 9, no. 5, p. 056016, 2012.

[9] K. Das and Z. Nenadic, "An efficient discriminant-based solution for small sample size problem," Pattern Recogn, vol. 42, no. 5, pp. $857-$ 866, 2009.

[10] K. Das and Z. Nenadic, "Approximate information discriminant analysis: A computationally simple heteroscedastic feature extraction technique," Pattern Recogn, vol. 41, no. 5, pp. 1548-1557, 2008.

[11] A. H. Do, P. T. Wang, C. E. King, S. N. Chun, and Z. Nenadic, "Brain-computer interface controlled robotic gait orthosis," J Neuroeng Rehabil, vol. 10, no. 1, p. 111, 2013.

[12] L. Wang, Y. Zhang, and J. Feng, "On the euclidean distance of images," IEEE Trans Pattern Anal Mach Intell, vol. 27, no. 8, pp. 13341339,2005

[13] S. A. Binder, C. B. Moll, and S. L. Wolf, "Evaluation of electromyographic biofeedback as an adjunct to therapeutic exercise in treating the lower extremities of hemiplegic patients.," Phys Ther, vol. 61, pp. 886-893, Jun 1981

[14] D. Dickson, K. Hollman-Gage, S. Ojofeitimi, and S. Bronner, "Comparison of functional ankle motion measures in modern dancers," Journal of Dance Medicine \& Science, vol. 16, no. 3, pp. 116-125, 2012. 